\title{
Exponentially Convergent Fourier-Chebshev Quadrature Schemes on Bounded and Infinite Intervals
}

\author{
John P. Boyd ${ }^{1}$
}

Received November 19, 1986

\begin{abstract}
The Clenshaw-Curtis method for numerical integration is extended to semiinfinite $([0, \infty]$ and infinite $[-\infty, \infty]$ intervals. The common framework for both these extensions and for integration on a finite interval is to (1) map the integration domain to $t \in[0, \pi]$, (2) compute a Fourier sine or cosine approximation to the transformd integrand via interpolation, and (3) integrate the approximation. The interpolation is most easily performed via the sine or cosine cardinal functions, which are discussed in the appendix. The algorithm is mathematically equivalent to expanding the integrand in (mapped or unmapped) Chebyshev polynomials as done by Clenshaw and Curtis, but the trigonometric approach simplifies the mechanics. Like Gaussian quadrature, the error for the change-of-coordinates Fourier method decreases exponentially with $N$, the number of grid points, but the generalized Curtis-Clenshaw algorithm is much easier to program than Gaussian quadrature because the abscissas and weights are given by simple, explicit formulas.
\end{abstract}

KEY WORDS: Quadrature; rational Chebyshev functions; adaptive quadrature; numerical integration.

\section{INTRODUCTION}

Clenshaw and Curtis (1960) introduced a simple method for evaluating integrals: expand the integrand $f(x)$ as a series of Chebyshev polynomials and then integrate the series term by term. Because the Chebyshev approximation converges exponentially fast with the number of terms, $N$, retained in the truncation, it follows that the accuracy of the Clenshaw-Curtis quadrature increases exponentially with $N$, too. This rate

\footnotetext{
${ }^{1}$ Department of Atmospheric and Oceanic Science, University of Michigan, Ann Arbor, Michigan 48109.
} 
of convergence is the same as that of Gaussian quadrature, but the accuracy is not quite as good: the Chebyshev method is exact when the integrand is a polynomial of degree $N$ whereas the $N$-point Gaussian algorithm is exact for polynomials of degree $2 N$. However, the quadrature weights and abcissas for the Chebyshev procedure are given by simple analytic formulas, whereas those for Gaussian quadrature must be stored in large tables, so the Chebyshev method is significantly easier to program, especially for large $N$.

The great strength of the Clenshaw-Curtis algorithm, however, is its suitability for adaptive quadrature. The authors note when $N$ is increased in Gaussian integration, it is necessary to recompute the integrand at each of the new abcissas. However, if the Chebyshev "trapezoidal rule" grid is employed, then whenever $N$ is doubled, half of the new grid points are identical with the old and all the previous function evaluations can be reused. In this context of $N$-doubling adaptation, the expand-inChebyshev-and-integrate method is clearly superior to Gaussian integration.

In this note, the Chebyshev integration method is extended from finite intervals to semi-infinite and infinite intervals. Because the mechanics of $N$-doubling adaption is adequately described in Clenshaw and Curtis (1960), we shall discuss only how to evaluate the integrals for fixed $N$, but we shall review integration on $x \in[-1,1]$ in order to discuss all the cases from a unified viewpoint.

The key idea in extending the Clenshaw-Curtis method to unbounded intervals is that of mapping: by making a change-of-coordinate, an infinite or semi-infinite interval may always be mapped into a finite one. However, we differ from Clenshaw and Curtis by mapping the integration onto the interval $[0, \pi]$ and expanding the integrand as a Fourier series instead of approximating the integrand as a Chebyshev series on $[-1,1]$. Because this transformation-to-a-Fourier problem has some advantages (explained below) even for integrals on a finite interval-Clenshaw-Curtis's original problem - we will discuss three separate integration ranges: $(1)[-1,1]$, (2) $[0, \infty]$, and (3) $[-\infty, \infty]$. Other intervals of integration can always be mapped into one of these three cases through a simple linear change of variable.

Section 2 offers an abstract overview of the method before the specific formulas for each of the three intervals is explained in Section 3. Since the convergence of these methods is intimately related to that of the underlying series, which in turn is discussed in Gottlieb and Orszag (1977), Voigt et al. (1985), and (for the unbounded intervals) Boyd (1987a, b), we shall leave the justification of these mappings to these earlier works. However, Section 4 does offer some brief illustrations of how these previously derived 
concepts apply to numerical integration. The final section is a summary. Previously unpublished formulas on the sine and cosine "cardinal" functions for trigonometric interpolation are collected in the Appendix.

\section{AN OVERVIEW OF INTEGRATION BY MAPPING AND EXPANSION}

The evaluation of the integral

$$
I \equiv \int_{-\infty}^{\infty} Q(y) d y
$$

has three main steps. The first is to change variables to the trigonometric coordinate, $t \in[0, \pi]$, via

$$
y \equiv f(t)
$$

where $f(t)$ is the mapping function. The integral becomes

$$
I \equiv \int_{0}^{\pi} Q(f[t]) f^{\prime}(t) d t
$$

where $f^{\prime}(t) \equiv d f / d t$.

The second step is to expand the integrand as a truncated Fourier series via trigonometric interpolation:

$$
q(t) \equiv Q(f[t]) f^{\prime}(t)=a_{0}+\sum_{n=1}^{N} a_{n} \cos (n t)+\sum_{n=1}^{N} b_{n} \sin (n t)
$$

Since the goal is numerical integration, this series form is not very useful; it is more convenient to combine the trigonometric basis functions into new functions $C_{j}(t)$ that are equal to 1 at the $j$ th interpolation point and vanish at all the others, than is,

$$
C_{j}\left(t_{i}\right)= \begin{cases}1, & i=j \\ 0, & i \neq j\end{cases}
$$

where the $t_{i}$ are the interpolation points.

There is no generally accepted name for the $C_{j}$ 's; by analogy with the Whittaker cardinal functions (Stenger, 1981), which in fact closely resemble the $C_{j}(t)$, we shall refer to the functions defined by $(2.5)$ as the "trigonometric cardinal functions."

The three changes-of-coordinate discussed in the next section give $q(t)$ which can be expanded as either a sine series $\left\{\right.$ all $a_{n}=0$; finite and semi- 
finite intervals $\}$ or a cosine series $\left\{b_{n}=0\right.$ for all $\left.n\right\}$. The sine and cosine cardinal functions are given in the Appendix.

Because of (2.5), the approximation to $q(t)$ becomes simply

$$
q(t) \equiv \sum_{j=1}^{N} q\left(t_{j}\right) C_{j}(t)
$$

The third and final step is to integrate $q(t)$ by integrating each of the cardinal functions. We obtain

$$
I=\sum_{j=1}^{N} w_{j} Q\left(y_{j}\right)
$$

where the quadrature abscissas are the images of the evenly spaced trigonometric grid under the mapping

$$
y_{j}=f\left(t_{j}\right), \quad j=1, \ldots, N
$$

and where the quadrature weights are defined by

$$
w_{j} \equiv f^{\prime}\left(t_{j}\right) \int_{0}^{\pi} C_{j}(t) d t
$$

In the next section, we discuss the implementation of tis common framework for each of the three classes of intervals.

\section{QUADRATURE MECHANICS}

Although there is more than one type of grid that gives exponential accuracy in trigonometric interpolation, we shall use the "trapezoidal rule" grid, which is defined by splitting the interval $t \in[0, \pi]$ into subintervals of length $1 / N$ and then placing the grid points at the boundaries of each subinterval. The reasons for this choice are that (1) the "trapezoidal" grid allows evaluations of the integrand to be reused when $N$ is doubled and (2) the grid points at $t=0$ and $\pi$ can usually be omitted since at those points $q(t)$ is always 0 in sine interpolation, and often 0 in cosine interpolation.

The cosine mapping used for the finite interval is simply the transformation that takes the Chebyshev polynomials in $y$ into the cosine functions in $t$ via the identity $T_{n}(y)=\cos (n t)$. Thus, our quadrature formula on $[-1,1]$ is identical with that of Clenshaw and Curtis (1960) even though the derivation is quite different. 
The mappings for the semi-infinite and infinite intervals are justified at length in Boyd (1986a, b), so without further ado the quadrature formulas are the following:

(1) $y \in[-1,1]$ :

$$
\begin{gathered}
y=\cos (t) \\
I_{1} \equiv \int_{-1}^{1} Q(y) d y=\int_{0}^{\pi} Q(\cos (t)) \sin (t) d t \\
\approx \sum_{i=1}^{N} w_{i} Q\left(\cos \left(t_{i}\right)\right) \\
t_{i} \equiv \pi i /(N+1), \quad i=1,2, \ldots, N \\
w_{i} \equiv \sin \left(t_{i}\right)[2 /(N+1)] \sum_{j=1}^{N} \sin \left(j t_{i}\right)[1-\cos (j \pi)] / j
\end{gathered}
$$

(2) $y \in[0, \infty]$ :

$$
\begin{gathered}
y=L \cot ^{2}(t / 2) \quad(L \text { is a user-chosen constant }) \\
I_{2} \equiv \int_{0}^{\infty} Q(y) d y=\int_{0}^{\pi} Q\left(L \cot ^{2}(t / 2)\right) 2 L \sin (t) d t /[1-\cos (t)]^{2} \\
\approx \sum_{i=1}^{N} w_{i} Q\left(L \cot ^{2}\left(t_{i} / 2\right)\right) \\
t_{i} \equiv \pi i /(N+1), \quad i=1, \ldots, N
\end{gathered}
$$$$
w_{i} \equiv\left\{2 L \sin \left(t_{i}\right) /\left[1-\cos \left(t_{i}\right)\right]^{2}\right\}[2 /(N+1)] \sum_{j=1}^{N} \sin \left(j t_{i}\right)[1-\cos (j \pi)] / j
$$

(3) $y \in[-\infty, \infty]$ :

$$
\begin{gathered}
y=L \cot (t) \quad(L \text { is a user-chosen constant }) \\
I_{3} \equiv \int_{-\infty}^{\infty} Q(y) d y \equiv \int_{0}^{\pi} Q(L \cot (t)) L d t / \sin ^{2}(t) \\
\approx \sum_{i=0}^{N+1} w_{i} Q\left(L \cot \left(t_{i}\right)\right) \\
w_{i}= \begin{cases}L \pi /\left[\sin ^{2}\left(t_{i}\right)(N+1)\right], & 0<i<(N+1) \\
L \pi /\left[\sin ^{2}\left(t_{i}\right)(2 N+2),\right] & i=0 \text { and } i=N+1\end{cases}
\end{gathered}
$$


The map constant $L$ for the unbounded intervals is $O(1)$ and may be optimized through trial and error or by applying the analytic estimates of Boyd (1982). The role of the map parameter is thoroughly discussed in Boyd (1986a, b).

As explained in these earlier works,each of these mappings normally transforms $Q(y)$ into a Fourier cosine series in $t$. [Certain exceptional cases are discussed in Boyd (1986a,b).] However, the change-of-integration factor, $f^{\prime}(t)$, introduces a subtlety.

For case (3), $y \in[-\infty, \infty]$, this factor is $1 / \sin ^{2}(t)$, which is symmetric about $t=0$. It follows that $q(t)$ for the infinite interval can be expanded as an exponentially convergent Fourier cosine series. The formulas for cosine interpolation then give (3.3e), which shows that the usual trapezoidal rule - only accurate to $O\left(1 / N^{2}\right)$ for general functions - has an error that decreases exponentially fast with $N$ when the integrand $q(t)$ is obtained by change-of-coordinate in an infinite integral.

For the other two cases, however, $f^{\prime}(t)$ is antisymmetric about $t=0$. This implies that $q(t)$ must be expanded as a Fourier sine series even though $Q(y(t))$ is a cosine series. The result is that we must use the more complicated weights shown in (3.1e) and (3.2e), which follow from integrating the sine cardinal functions. The trapezoidal rule is only accurate to $O\left(1 / N^{2}\right)$ for these two cases, but we can achieve exponential accuracy for the same grid points merely by changing the weight functions $w_{i}$.

One other subtlety is that for the unbounded intervals, $Q(y)$ must decrease sufficiently fast as $y \rightarrow \infty$ so that $q(t)$ is bounded at $t=0$ and $t=\pi$. [The integral may still be well defined even if $q(t)$ is unbounded at these end points, but the accuracy will not decrease exponentially with $N$.] This is more restrictive than the conditions for $Q(y)$ to be expandable in terms of the (mapped) Fourier cosine functions (Boyd, 1986a, b). Thus, $\tanh (y)$ is approximated by the first 20 odd cosines to within a maximum absolute error of $10^{-8}$ on $y \in[-\infty, \infty]$ with the map (3.3a) with $L=2$, but the corresponding quadrature scheme fails because $q(t)$ is singular as $1 / t^{2}$ as $t \rightarrow 0$. We can sum up this requirement as the following:

Theorem. A necessary (but not sufficient) condition for the mapped Fourier quadrature scheme to converge exponentially fast with $N$, the number of grid points is that

(a) $Q(y)$ decreases as $O\left(y^{-3 / 2}\right)$ as $y \rightarrow \infty ; y \in[0, \infty]$

(b) $Q(y)$ decrease as $O\left(y^{-2}\right)$ as $|y| \rightarrow \infty ; y \in[-\infty, \infty]$

Proof. Taylor expansion of $q(t)\left[\equiv Q(f(t)) f^{\prime}(t)\right]$ about $t=0$ and $t=\pi$. 
The final comment on the mechanics of quadrature is that the formulas for integration on $[-\infty, \infty]$ have been written in terms of $N+2$ points because one usually omits the end points at $t=0$ and $t=\pi$. The sole exception is when $Q(y)$ decreases as $1 / y^{2}$ precisely: if $Q(y)$ decays more rapidly, then these points do not contribute to the integral, and if $Q(y)$ decays more slowly, the integral in $t$ is unbounded at the endpoints.

\section{NUMERICAL EXAMPLES: ILLUSTRATIONS OF ASYMPTOTIC BEHAVIOR}

The theory of Boyd (1982) shows that the asymptotic Fourier coefficients of a function $q(t)$ which is derived via a change of coordinates from a function $Q(y)$ on an infinite or semi-infinite interval is the sum of two distinct types of terms: (1) residues from poles of $Q(y)$ and similar branch point contributions and (2) steepest descent terms that depend on how rapidly the function decays as $y \rightarrow \infty$. Figures 1 and 2 show the decay of error for the integrals of two simple functions on $[-\infty, \infty]$ which each have only one type of term. The function

$$
Q(y)=1 /\left(1+y^{2}\right)
$$

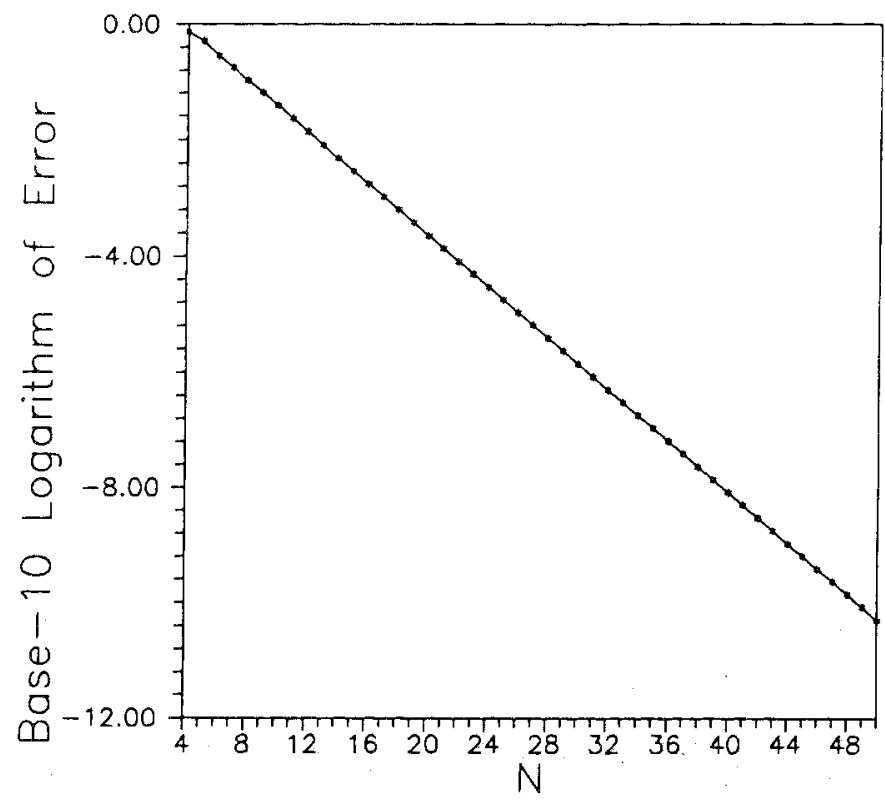

Fig. 1. Base-10 logarithm of the absolute vaiue of the absolute error versus $N$, the number of grid points, for the Fourier quadrature of $Q(y)=1 /\left(1+y^{2}\right)$ on $y \in[-\infty, \infty]$. 


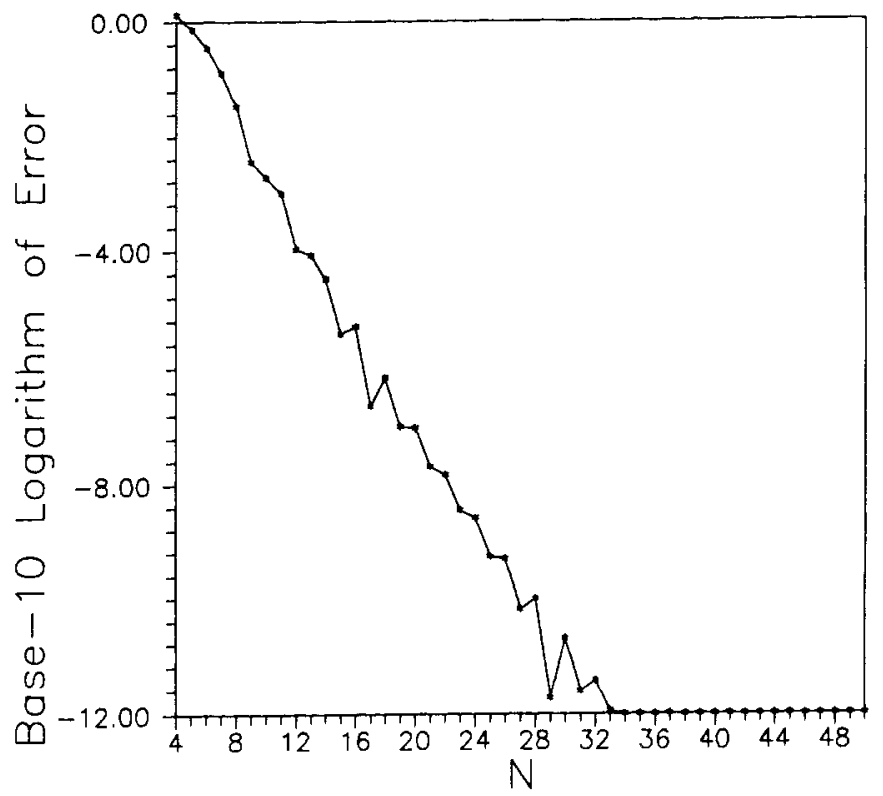

Fig. 2. Base-10 logarithm of the absolute value of the absolute error versus $N$, the number of grid points, for the Fourier quadrature of $Q(y)=\exp \left(-y^{2}\right)$ on $y \in[-\infty, \infty]$.

decays algebraically rather than exponentially with $y$, so its asymptotic Fourier coefficients are determined by the residues of the poles at $y= \pm i$. As explained in Boyd (1986a), this implies that the quadrature error will decrease geometrically with $N$, that is,

$$
\log \left|E_{N}\right| \sim-N d, \quad N \rightarrow \infty
$$

On the log-linear plot in Fig. 1, the predicted error decay is a straight line exactly as observed. Because the singularities are on the imaginary axis, the decay is monotonic, but the error would be modulated by an oscillatory phase if the singularities were elsewhere.

The function

$$
Q(y)=\exp \left(-y^{2}\right)
$$

in contrast, has no singularities; its asymptotic behavior is controlled by a steepest descent term, which in turn is dictated by the exponential decay as $|y| \rightarrow \infty$ for real $y$. (Equivalently, the steepest descent terms are associated with the essential singularities at $t=0, \pi$, the images of $y= \pm \infty$ under the 
change of coordinate.) The error decreases subgeometrically in the sense that $r<1$ in the asymptotic estimate

$$
\log \left|E_{N}\right| \sim-d^{\prime} N^{r}+\log \left[\cos \left(d^{\prime \prime} N^{r}+\phi\right]\right.
$$

where $d^{\prime}, d^{\prime \prime}, \phi$, and $r$ are all constants ( $r=2 / 3$ for the Gaussian). Figure 2 illustrates both the oscillations induced by the cosine term in (4.4) and the gradual decrease in the (mean) slope as $N$ increases.

When the asymptotic Fourier coefficients contain both types of terms, the steepest descent contribution must always dominate for sufficiently large $N$ and the asymptotic convergence is subgeometric. Figure 3 shows, however, that for

$$
f(y)=\operatorname{sech}(y)
$$

the convergence is geometric - a straight line-for $N \leqslant 16$ because the Fourier coefficients for small $N$ are dominated by the poles of $\operatorname{sech}(y)$ at $y= \pm i \pi / 2$. Only if one is interested in more than four decimal places of accuracy is it necessary to go to larger $N$ where the coefficients are cominated by the more slowly decreasing steepest descent (end-point) terms.

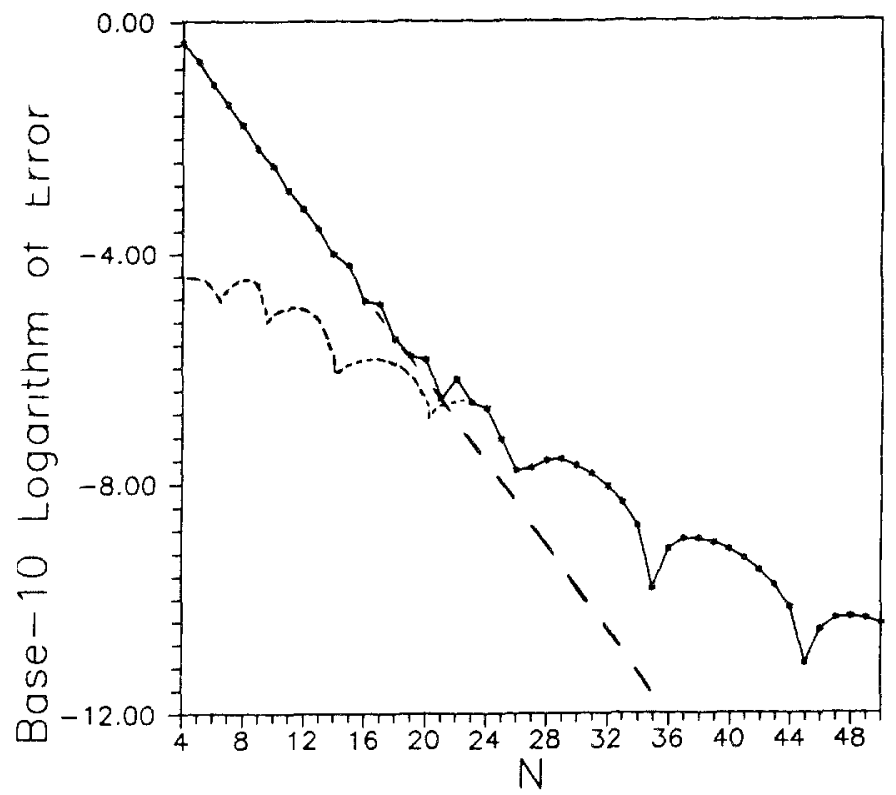

Fig. 3. Base-10 logarithm of the absolute value of the absolute error versus $N$, the number of grid points, for the Fourier quadrature of $Q(y)=\operatorname{sech}(y)$. Solid curve: the actual quadrature error. Dashed line: schematic of the contribution of the poles at $y= \pm i \pi / 2$. Dotted curve: schematic of the contribution from the end points (steepest descent term). 
Because of this competition between these two classes of terms that sum to give the asymptotic Fourier coefficients, it is difficult to apply the rules of Boyd (1982) to all situations. Experimentation will probably be needed both to optimize the map parameter $L$ and estimate the error. The consolations are that (1) accuracy is rather insensitive to $L$ and (2) the exponential convergence with $N$ makes it easy to integrate to many decimal places - and be sure of high precision — even with a poor choice of $L$ and a relatively small number of grid points.

\section{SUMMARY}

In this article, we have extended the Clenshaw-Curtis quadrature scheme to semi-infinite and infinite intervals. Although the method is not quite as accurate as Gaussian quadrature for a given number of grid points, it does offer two important advantages. First, the change-of-coordinate Fourier method has simple, explicit abscissas and weights. In contrast, the Gaussian quadrature abscissas are the irrational roots of polynomials, and must be stored in tables or recalculated by root-solving for each choice of $N$. Second, the Clenshaw-Curtis procedure is more efficient for adaptive quadrature because previous evaluations of the integrand $Q(y)$ can be reused when $N$ is doubled.

Like Gaussian quadrature, the Fourier method has a accuracy that increases exponentially fast with the number of grid points. Because of this and because it is so simple to program, the change-of-coordinate Fourier algorithm is a good integration method for both bounded and unbounded intervals.

\section{ACKNOWLEDGMENTS}

This work was supported by grant No. OCE8509923 from the National Science Foundation. I thank Warren Smith for helpful discussions.

\section{APPENDIX: CARDINAL FUNCTIONS FOR SINE AND COSINE INTERPOLATION}

The review by Gottlieb, Hussaini, and Orszag in Voigt et al. (1984) lists the cardinal functions for general trigonometric interpolation, but the cardinal functions for sine interpolation (of functions antisymmetric about $t=0)$ and cosine interpolation [ $q(t)$ symmetric about the origin] do not seem to be readily accessible. These specialized cardinal functions can be derived from the general trigonometric cardinal functions by combining the 
later in pairs: the symmetry of $q(t)$ for cosine interpolation implies that the coefficients of the general cardinal functions $C_{j}(x)$ and $C_{-j}(x)$ are identical, for example. Through elementary algebra and trigonometric identities we find

$$
\begin{aligned}
C_{j}^{\cos }(t) & =(-1)^{j+1} \sin (t) \sin ([N+1) t) /\left\{c_{j}(N+1)\left[\cos (t)-\cos \left(t_{j}\right)\right]\right\} \\
& =\left\{2 /\left[(N+1) c_{j}\right]\right\}\left\{\sum_{m=0}^{N+1} \cos \left(m t_{j}\right) \cos (m t) / c_{m}\right\} \\
C_{j}^{\sin }(t) & =(-1)^{j+1} \sin \left(t_{j}\right) \sin [(N+1) t] /\left\{(N+1)\left[\cos (t)-\cos \left(t_{j}\right)\right]\right\} \\
& =(2 /[N+1]) \sum_{m=1}^{N} \sin \left(m t_{j}\right) \sin (m t)
\end{aligned}
$$

where the grid points are

$$
\begin{gathered}
t_{i}=\pi i /(N+1), \quad i=0, \ldots, N+1 \quad \text { (cosine interpolation) } \\
i=1, \ldots, N \quad \text { (sine interpolation) } \\
c_{j}= \begin{cases}2 & \text { if } j=0 \text { or } j=N+1 \\
1 & \text { if } 0<j<(N+1)\end{cases}
\end{gathered}
$$

Two forms are given for each cardinal function because the first expression makes it easy to verify that $C_{j}\left(t_{i}\right)=0$ if $i \neq j$ and $C_{j}\left(t_{j}\right)=1$ while the second version shows that each cardinal function is a linear combination of the original sine or cosine basis functions.

\section{REFERENCES}

Boyd, J. P. (1982). The optimization of convergence for Chebyshev polynomial methods in an unbounded domain. J. Comput. Phys. 45, 43-79.

Boyd, J. P. (1987a). Spectral methods using rational basis functions on an infinite interval. J. Comput. Phys. 69, 112-142.

Boyd, J. P. (1987b). Orthogonal rational functions on a semi-infinite interval. J. Comput. Phys. 70, 63-88.

Clenshaw, C. W., and Curtis, A. R. (1960). A method for numerical integration on an automatic computer. Numer. Math. 2, 197-205.

Gottlieb, D, and Orszag, S. A. (1977). Numerical Analysis of Spectral Methods. SIAM, Philadelphia, $172 \mathrm{pp}$.

Stenger, F. (1981). Numerical methods based on Whittaker cardinal, or sinc functions. SIAM Rev. 23, 165-224.

Voigt, R. G., Gottlieb, D., and Hussaini, M. Y., eds., 1984: Spectral Methods for Partial Differential Equations. SIAM, Philadelphia, 265 pp. 